\title{
Signatures of Moat Regimes in Heavy-Ion Collisions
}

\author{
Robert D. Pisarski* \\ Physics Department, Brookhaven National Laboratory, Upton, New York 11973, USA \\ Fabian Rennecke® $\oplus^{\dagger}$ \\ Condensed Matter Physics and Materials Science Division, Brookhaven National Laboratory, Upton, New York 11973, USA
}

(Received 23 March 2021; revised 9 June 2021; accepted 8 September 2021; published 5 October 2021; corrected 3 March 2022)

\begin{abstract}
Heavy-ion collisions at small beam energies have the potential to reveal the rich phase structure of QCD at low temperature and nonzero density. In this case spatially modulated regimes with a "moat" spectrum can arise, where the minimum of the energy is over a sphere at nonzero momentum. We show that if the matter created in heavy-ion collisions freezes out in such a regime, particle numbers and their correlations peak at nonzero, instead of zero, momentum. This effect is much more dramatic for multiparticle correlations than for single-particle spectra. Our results can serve as a first guideline for a systematic search of spatially modulated phases in heavy-ion collisions.
\end{abstract}

DOI: 10.1103/PhysRevLett.127.152302

Introduction.-The collisions of heavy ions at high energy, such as at the Relativistic Heavy Ion Collider at BNL and the Large Hadron Collider at CERN, have demonstrated experimentally the production of a qualitatively new state of matter. This is possibly the transition to a quark-gluon plasma at a pseudocritical temperature $T \approx 154 \mathrm{MeV}$ and net baryon chemical potential $\mu_{B} \approx 0$. As the energy of the collision decreases, one probes the phase diagram at lower $T$ and larger $\mu_{B}[1,2]$.

Model studies suggest that regimes with periodic spatial modulations can occur at high $\mu_{B}$. There, particles can have a "moat" spectrum, where the minimum of the energy is not at zero momentum, but lies over a sphere at non zero spatial momentum. If the energy at the bottom of the moat is zero, an instability toward the formation of spatially inhomogeneous condensates might occur [3]. Studies in $1+1$ dimensional models that are exactly soluble as the number of flavor $N_{f} \rightarrow \infty$ have shown that inhomogeneous chiral condensates always arise at low temperature and large density [4-6]. How the moat spectrum manifests itself depends upon the internal symmetry. For these soluble models the global symmetry is either discrete $[Z(2)]$ or $O(2)$, and the spatially inhomogeneous condensate derived at $N_{f}=\infty$ should persist at finite $N_{f}$ [7].

Recent analysis, however, suggests that this is radically changed by the presence of Goldstone bosons. Consider an $O(N)$ model, so that when $N>2$, the spatially

Published by the American Physical Society under the terms of the Creative Commons Attribution 4.0 International license. Further distribution of this work must maintain attribution to the author(s) and the published article's title, journal citation, and DOI. Funded by SCOAP. inhomogeneous condensate (or "chiral spiral") has $N-2$ Goldstone modes. About the (simplest) chiral spiral, unexpectedly the Goldstone bosons have zero energy at the bottom of the moat. Since this occurs at nonzero momentum, it is a double pole, and generates severe linear infrared divergences [8]. A solution at infinite $N$ demonstrates that a dynamical mass for the Goldstone bosons is generated at the bottom of the moat, and disorders the would-be chiral spirals into a type of quantum pion liquid $(\mathrm{Q} \pi \mathrm{L})[10-13]$. This is a disordered phase, where unlike a chiral spiral, there is no preferred direction. Because the minimum of the energy is at nonzero momentum, it is still associated with periodic spatial modulations. For QCD, where two light flavors are described by an $O(4)$ model, this suggests that instead of a condensate of chiral spirals, there is a $\mathrm{Q} \pi \mathrm{L}[14]$.

In this Letter we consider the experimental signatures of a moat spectrum. The difference between a normal homogeneous phase, where the smallest energy is at zero momentum, and for a moat regime, where it is at nonzero momentum, should be direct. Certainly the particle distribution with a moat spectrum should be peaked at nonzero momentum [10]. We argue that this elementary feature can leave distinctive signatures in the production of particles and their correlations, which are measurable in heavy-ion collisions.

Both in QCD [15] and in models [3-6] a moat spectrum can arise in a large region of the phase diagram. Thus, the matter created in heavy-ion collisions at low beam energies is likely to traverse a moat regime if it exists at large $\mu_{B}$. For simplicity, we assume that freeze-out occurs in this region. This does not exclude the possibility that the signatures discussed here could also occur if the system is in a moat regime at earlier stages of its evolution. In any case, if 
particles at sufficiently low momenta can be measured, we predict a peak in the momentum dependence of particle numbers and correlations at nonzero momentum. This is a clear signature of spatial modulations in dense QCD, and could signal either a $\mathrm{Q} \pi \mathrm{L}$ or a precursor to the formation of an inhomogeneous phase.

Moat spectrum.-We focus on the subsector of the QCD action at low energy which contains quark bilinears, and is described by an $O(N)$ field $\vec{\phi}$; for $N=4$, this contains the $\sigma$ meson and three pions for two light flavors:

$\mathcal{L}=\frac{1}{2}\left(\partial_{0} \vec{\phi}\right)^{2}-\frac{Z}{2}\left(\partial_{i} \vec{\phi}\right)^{2}-\frac{W}{2}\left(\partial_{i}^{2} \vec{\phi}\right)^{2}-\frac{m_{\text {eff }}^{2}}{2} \vec{\phi}^{2}+\cdots$

There are higher order terms in the Lagrangian, such as a quartic coupling $\sim\left(\vec{\phi}^{2}\right)^{2}$, but all that matters for our analysis are the terms quadratic in $\vec{\phi}$. In vacuum, by Lorentz invariance $Z=1$, but a medium is not Lorentz invariant, and so $Z$ can be less than unity. In a moat regime, one has the more dramatic feature that $Z$ is negative; then terms of quartic order in the spatial momentum are required for stability at large momentum. As an effective theory at low momenta, we therefore add such a term, whose coefficient, $W$, must be positive.

If $Z<0$, then classically an instability toward the formation of an inhomogeneous condensate, e.g., a chiral spiral $\vec{\phi}=\sigma_{0}\left[\cos \left(p_{\min } z\right), \sin \left(p_{\min } z\right), 0, \ldots, 0\right]$, can occur, where $p_{\min }^{2}$ is the bottom of the moat. However, for $N \rightarrow \infty$ it can be shown rigorously that a double pole in the Goldstone modes at $p_{\min }$ always disorders the inhomogeneous condensate [11-13]. Instead of such condensate, the system is in a $\mathrm{Q} \pi \mathrm{L}$ phase, which is characterized by spatial correlations of the field $\phi$ which fall off with the typical exponential times an oscillatory function. While this is certain at large $N$, the $\mathrm{Q} \pi \mathrm{L}$ should arise whenever there are Goldstone bosons for a chiral spiral, $N>2$ [11], and certainly includes $N=4$.

In the moat regime, $Z<0$, and the energy

$$
E_{\phi}\left(\mathbf{p}^{2}\right)=\sqrt{Z \mathbf{p}^{2}+W\left(\mathbf{p}^{2}\right)^{2}+m_{\mathrm{eff}}^{2}},
$$

has a minimum at nonzero momentum, $p_{\min }^{2}=-Z /(2 W)$. $p_{\min }$ is related to the wave number of the underlying spatial modulation. Examples for different $Z$ are shown in Fig. 1. We note that for sufficiently large momenta, the energy should reduce to the one in vacuum. Admittedly, the values which we choose in Fig. 1 and henceforth are illustrative and do not follow from a detailed model. However, the results of [15] indicate that deeper in the moat regime $Z$ could even take much larger negative values than the ones explored here [16]. More importantly, it is natural to expect that $p_{\min }$ is related to a low-energy scale $\lesssim \Lambda_{\mathrm{QCD}}$. We further require $E_{\phi}\left(\mathbf{p}^{2}\right)>0$ for all $\mathbf{p}^{2}$ in order to avoid an instability. In any case, our analysis builds on the intuitive

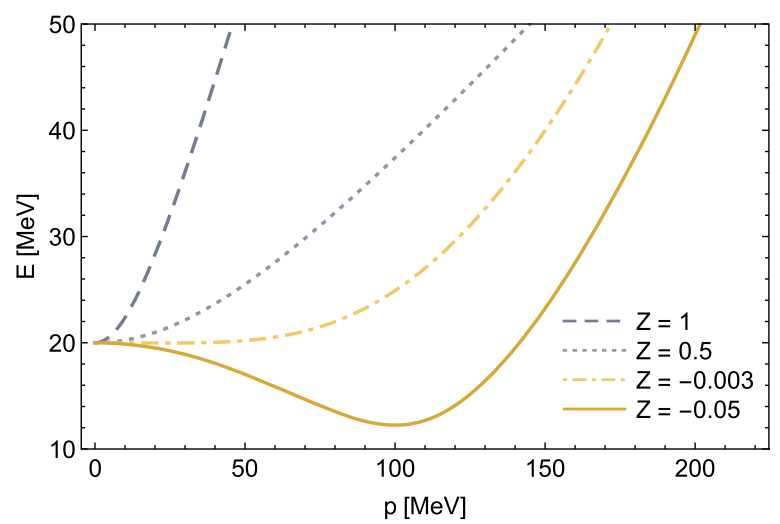

FIG. 1. Dispersion relations in the normal ( $Z>0$, gray lines) and moat $(Z<0$, yellow lines) regime as functions of the spatial momentum. As opposed to the normal phase, the energy is minimal at a finite momentum in the moat regime. We have chosen $W=2.5 \mathrm{GeV}^{-2}$ when $Z<0$.

fact that when $p_{\min }$ is nonzero, the production of particles with nonzero momentum is favored.

Particle spectra.-We consider the production of particles in heavy-ion collisions in more detail. Matter produced in heavy-ion collisions expands rapidly and cools until the system is so dilute that particles freeze out: elastic and inelastic scattering cease and hadrons stream to the detector. Hence, we need to describe particles on the freezeout surface, where, if the system passes through a moat regime during its expansion, particles can carry signatures of this regime. Here, we restrict ourselves to the case where the freeze-out occurs in the moat regime. Studying the case where the system traverses a moat regime during earlier stages of its evolution, but freezes out in a different phase, is beyond the scope of the present work.

The number of particles on the freeze-out surface can be computed by using the formula of Cooper and Frye [17]. This assumes free (quasi) particles with an ordinary dispersion relation, $\left(p^{0}\right)^{2}=\mathbf{p}^{2}+m^{2}$. Here we need to generalize this result to describe particles with an arbitrary dispersion relation [18]; see Refs. [19-21] for related discussions. In thermal equilibrium, the Wigner function $F_{\phi}(p)$ of the field $\phi$ is proportional to the spectral function $\rho_{\phi}$,

$$
F_{\phi}(p)=2 \pi \rho_{\phi}\left(p^{0}, \mathbf{p}\right) n_{B}\left(p_{0}\right),
$$

where $n_{B}\left(p_{0}\right)$ is the Bose-Einstein statistical distribution function. With this, the momentum dependent spectrum of $\phi$ on the freeze-out surface for particle $i$ is given by

$n_{i} \equiv \frac{d^{3} N_{\phi}}{d \mathbf{p}_{i}^{3}}=\frac{2}{(2 \pi)^{3}} \int_{\Sigma} d \Sigma_{\mu} \int \frac{d p_{i}^{0}}{2 \pi} p_{i}^{\mu} \Theta\left(\breve{p}_{i}^{0}\right) F_{\phi}\left(\breve{p}_{i}\right)$.

$\Sigma$ is the freeze-out surface, which is given by a threedimensional submanifold of four-dimensional spacetime, 
determined by the hypersurface with a fixed temperature, the freeze-out temperature $T_{f}$. The coordinates on this surface are $w^{1,2,3}$, and the induced metric $G_{i j}=$ $g^{\mu \nu}\left(d x^{\mu} / d w^{i}\right)\left(d x^{\nu} / d w^{j}\right)$, where $g^{\mu \nu}=\operatorname{diag}(1,-1,-1,-1)$ is the Minkowski metric. The integral measure over the hypersurface is therefore given by $d \Sigma^{\mu}=\hat{v}^{\mu} \sqrt{|\operatorname{det} G|} d^{3} w$, with the normal vector $\hat{v}^{\mu}=v^{\mu} /|v|$ given by the generalized cross product of the tangent vectors on $\Sigma$, $v^{\mu}=\bar{\epsilon}^{\mu \alpha \beta \gamma}\left(d x_{\alpha} / d w^{1}\right)\left(d x_{\beta} / d w^{2}\right)\left(d x_{\gamma} / d w^{3}\right)$, where $\bar{\epsilon}^{\mu \alpha \beta \gamma}$ is the covariant Levi-Civita tensor.

The frequency $\breve{p}_{0}$ and the spatial momentum $\breve{\mathbf{p}}$ are boosted by the fluid four-velocity $u^{\mu}$ of the medium at freeze-out, $\breve{p}_{0}=u^{\mu} p_{\mu}$ and $\breve{\mathbf{p}}^{2}=\Delta^{\mu \nu} p_{\mu} p_{\nu}$, where $\Delta^{\mu \nu}=$ $u^{\mu} u^{\nu}-g^{\mu \nu}$ is the projection operator for spatial directions transverse to $u^{\mu}$. Note that $F_{\phi}(\breve{p})=2 \pi \rho_{\phi}\left(\breve{p}_{0}, \breve{p}^{2}\right) n_{B}\left(\breve{p}_{0}\right)$, so that the spectral function and the thermal distribution are evaluated at the boosted momenta. The Heaviside function $\Theta\left(\breve{p}_{0}\right)$ ensures that only particles with positive energy contribute. We implicitly assumed rotational invariance of the spectral function, since it is not broken in the moat regime, although Eq. (4) is valid regardless.

Eq. (4) is obtained by integrating the covariant particle number current over the freeze-out surface. If the spectral function of the particle is known, for a given parametrization of the freeze-out surface and the fluid velocity, particle spectra can readily be computed. The freeze-out surface and the fluid velocity are obtained from the hydrodynamic evolution of the system. For simplicity we parametrize ideal hydrodynamics in terms of a boost-invariant blastwave model [22,23], assuming that the velocity of the radial expansion of the system grows proportional to the radial distance, and that freeze-out happens at a fixed proper time $\tau_{f}$. The freeze-out curve can then be described in terms of the longitudinal rapidity $\eta_{\|}=\operatorname{artanh}(z / t)$, the radius $r=\sqrt{x^{2}+y^{2}}$, and the angle $\phi=\arctan (y / x)$, where $z$ is the direction of the beam. We use the parametrization of Ref. [24], which yields for the fluid velocity

$$
u^{\mu}=\left(u^{\tau} \cosh \eta_{\|}, u^{r} \cos \phi, u^{r} \sin \phi, u^{\tau} \sinh \eta_{\|}\right),
$$

with the radial flow velocity $u^{r}=\bar{u}(r / \bar{R}) \Theta(\bar{R}-r)$ and $u^{\tau}=\sqrt{1+\left(u^{r}\right)^{2}}$. The fluid velocity has two free parameters, the radial velocity $\bar{u}$ and the fireball radius $\bar{R}$. The vector normal to the freeze-out surface is $\hat{v}^{\mu}=\left(\cosh \eta_{\|}, 0,0, \sinh \eta_{\|}\right)$, and the determinant of the induced metric is $\sqrt{|\operatorname{det} G|}=\tau_{f} r \Theta(\bar{R}-r)$.

The relevant physics of the moat regime is encoded in the spectral function $\rho_{\phi}\left(p^{0}, \mathbf{p}\right)$. While we use a boost-invariant blast-wave model for the fluid-dynamical part of our calculation, particles with a moat spectrum clearly break boost invariance. We compute the quasiparticle spectral function from the free Lagrangian in Eq. (1),

$$
\rho_{\phi}\left(\breve{p}_{0}, \breve{\mathbf{p}}^{2}\right)=\operatorname{sign}\left(\breve{p}_{0}\right) \delta\left[\left(\breve{p}_{0}\right)^{2}-E_{\phi}^{2}\left(\breve{\mathbf{p}}^{2}\right)\right],
$$

where $E_{\phi}$ is the energy given in Eq. (2). Note that since the energy is quartic in the spatial momenta, performing the frequency integration in Eq. (4) even with this simple spectral function is nontrivial. The momenta in Eq. (6) are boosted, so the on shell condition $\left(\breve{p}_{0}\right)^{2}=E_{\phi}^{2}\left(\breve{\mathbf{p}}^{2}\right)$ is in general a quartic equation in the rest frame momentum $p_{0}$. Of the four possible complex-valued solutions, only the ones which give real, positive $\breve{p}_{0}$ contribute to Eq. (4). For ordinary free bosons, i.e., with $Z=1$ and $W=0$ in Eq. (1), this computation is trivial and Eq. (4) reduces to the wellknown Cooper-Frye formula.

The spectral function in Eq. (6) implies that the smallest energy $\breve{p}_{0}$ that contributes to the spectrum is given by the minimum of $E_{\phi}^{2}\left(\breve{\mathbf{p}}^{2}\right)$. In the moat regime this minimum is at a nonzero spatial momentum. Since the thermal distribution function gives the largest contribution at the lowest energy in Eq. (4), most particles are produced at nonzero momentum.

As shown in Fig. 21 of Ref. [15], there are large regions in the $T-\mu_{B}$ plane in which the wave function renormalization $Z$ is negative, indicating a moat regime such as the $\mathrm{Q} \pi \mathrm{L}$. The parameters $Z, W$, and $m_{\mathrm{eff}}$, and thus the spectral function, are all functions of $T$ and $\mu_{B}$. These can be computed from first principles by methods such as the functional renormalization group following Refs. [15] and, e.g., [25-27]. For illustrative purposes, we choose parameters where the main features of the particle spectrum are apparent. In the homogeneous phase we choose $W=0$ and $Z>0$, while in the moat regime we take $W=2.5 \mathrm{GeV}^{-2}$ and $Z<0$. In both cases we take $m_{\text {eff }}=20 \mathrm{MeV}$. We stress again that these are illustrative, yet reasonable parameters, as explained above.

To fix the thermodynamic and blast-wave parameters, we first pick a beam energy of $\sqrt{s}=5 \mathrm{GeV}$, since it yields a system which is dense enough to traverse the preliminary moat region in Ref. [15]. The corresponding freeze-out temperature and chemical potential from the statistical model fits in Ref. [28] are $T_{f}=115 \mathrm{MeV}$ and $\mu_{B, f}=$ $536 \mathrm{MeV}$. From blast-wave fits to experimental data at different beam energies in Ref. [29], we read off the blastwave parameters $\bar{u}=0.3, \bar{R}=8 \mathrm{fm}$, and $\tau_{f}=5 \mathrm{fm} / \mathrm{c}$.

Having fixed all unknowns in Eq. (4), we show the resulting particle spectra for different $Z$ in Fig. 2 as a function of the transverse momentum $p_{T}=\sqrt{p_{x}^{2}+p_{y}^{2}}$. By rotational invariance we can set the azimuthal momentum angle $\phi_{p}=\arctan \left(p_{y} / p_{x}\right)=0$, and take the momentum space rapidity $y=\operatorname{arctanh}\left(p_{z} / p_{0}\right)=0$. The numerical results show the behavior anticipated above. Since as a function of momentum the energy is shallower for smaller $|Z|$, so are the spectra. Further, in the moat regime the minimal energy, $E_{\min }=\sqrt{m_{\text {eff }}^{2}-Z^{2} / 4 W}$, is less than the 


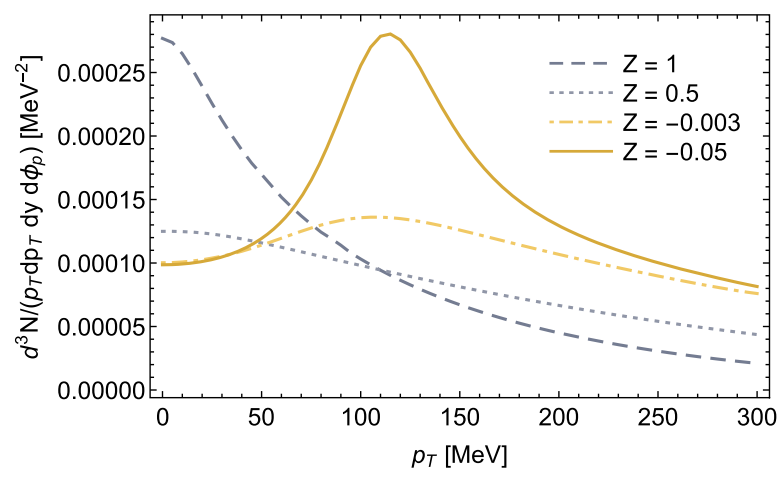

FIG. 2. Comparison between the transverse momentum spectra in the normal ( $Z>0$, gray lines) and moat $(Z<0$, yellow lines) regime. While most particles are produced at zero momentum and the spectrum decays exponentially with $p_{T}$ in the normal phase, it has a distinct maximum at nonzero $p_{T}$ in the moat regime.

minimal energy in the normal phase $m_{\text {eff }}$. Thus, as $Z$ becomes more negative, the energy is lowered and particle production is increased at nonzero momentum. Since the radial boost velocity increases toward the edges of the fireball, the $p_{T}$ spectrum shows a slightly washed-out peak at finite momentum corresponding to a boosted $p_{\min }$. We expect that the moat dispersion eventually turns into the normal one at large momenta. Since this is not captured in our model, the large $p_{T}$ behavior should not be taken too seriously here.

In summary, a monotonously increasing $p_{T}$ spectrum at low momenta, and a peak at finite momentum, are clear signatures of a moat regime.

Particle number correlations. - In addition to the particle spectra, a moat spectrum should also affect the momentum dependence of correlations in particle number. It is reasonable to anticipate that correlations are even more sensitive to moat regimes than the spectra. These can be computed just by considering single-particle correlations, since while there is no long-range order in the system, the particles are modified by their propagation in the medium. This is in contrast to a critical endpoint, where long-range correlations, and hence genuine multiparticle effects, appear.

Particle number correlations are given by a straightforward generalization of Eq. (4),

$$
\begin{aligned}
\left\langle\prod_{i} \frac{d^{3} N_{\phi}}{d \mathbf{p}_{i}^{3}}\right\rangle= & {\left[\prod_{i} \frac{2}{(2 \pi)^{3}} \int d \Sigma_{i}^{\mu} \int \frac{d p_{i}^{0}}{2 \pi}\left(p_{i}\right)_{\mu} \Theta\left(\breve{p}_{i}^{0}\right)\right] } \\
& \times\left\langle\prod_{i} F_{\phi}\left(\breve{p}_{i}\right)\right\rangle .
\end{aligned}
$$

Nontrivial correlations arise when $F_{\phi}$ fluctuates. Here, we focus on thermodynamic fluctuations in the spacetimedependent temperature, baryon chemical potential, and fluid velocity of the expanding medium. These are summarized by the quantity

$$
\kappa_{i}^{\mu}(x)=\left[T(x), \mu_{B}(x), u^{\mu}(x)\right]_{i} .
$$

$\kappa$ fluctuates around its mean value, $\langle\kappa\rangle=\bar{\kappa}$, i.e., $\kappa=$ $\bar{\kappa}+\delta \kappa$, which implies $\langle\delta \kappa\rangle=0$. Fluctuations in $\kappa$ induce those in $F_{\phi}$. Assuming that the fluctuations are small, $F_{\phi}$ can be expanded in powers of $\delta \kappa$, and correlations of Wigner functions translate directly into correlations of thermodynamic parameters. For example, expanding up to second order in $\delta \kappa$, the connected two-point correlation of $F_{\phi}$ is

$$
\left\langle F_{\phi} F_{\phi}\right\rangle_{c}=\left.\frac{\partial F_{\phi}}{\partial \kappa_{i}^{\mu}} \frac{\partial F_{\phi}}{\partial \kappa_{j}^{\nu}}\right|_{\bar{\kappa}}\left\langle\delta \kappa_{i}^{\mu} \delta \kappa_{j}^{\nu}\right\rangle+\mathcal{O}\left(\delta \kappa^{3}\right),
$$

where we used that the connected two-point function is determined only by the fluctuations, $\left\langle F_{\phi}^{2}\right\rangle_{c}=\left\langle F_{\phi}^{2}\right\rangle-\left\langle F_{\phi}\right\rangle^{2}$.

To compute the thermodynamic average, we generalize the classic treatment of thermodynamic fluctuations in Ref. [9], using unpublished results of Ref. [30]. We construct the generating functional for thermodynamic correlations on the freeze-out surface,

$e^{W[J]}=\int \mathcal{D} \kappa \exp \int d \Sigma_{\mu}\left[\Delta s^{\mu}(w)+J(w)_{i \nu} \hat{v}^{\mu} \delta \kappa_{i}^{\nu}(w)\right]$.

Taking $n$ derivatives of $W[J]$ with respect to the source $J$ and then setting $J=0$ yields the connected $n$-point function $\sim\left\langle(\delta \kappa)^{n}\right\rangle_{c} . \Delta s^{\mu}$ is the change in the entropy current density due to fluctuations in each fluid cell on the freeze-out surface, i.e., the difference between the entropy current density of the subsystem away from equilibrium specified by $\bar{\kappa}+\delta \kappa$ and that in equilibrium at $\bar{\kappa}$. For small fluctuations, it is given by the second-order correction of the expansion of the entropy current in terms of the thermodynamic fields,

$$
\Delta s^{\mu}=\left.\frac{1}{2} \frac{\partial^{2} s^{\mu}}{\partial \kappa_{i}^{\nu} \partial \kappa_{j}^{\rho}}\right|_{\bar{\kappa}} \delta \kappa_{i}^{\nu} \delta \kappa_{j}^{\rho} .
$$

Using the covariant form of the Euler relation, $d s^{\mu}=$ $\left(u_{\nu} / T\right) d T^{\mu \nu}-\left(\mu_{B} / T\right) d n^{\mu}$, where $T^{\mu \nu}=\epsilon u^{\mu} u^{\nu}+p \Delta^{\mu \nu}$ is the ideal energy-momentum tensor, we find

$$
\hat{v}_{\mu} \Delta s^{\mu}=-\frac{1}{2} \delta \kappa_{i \mu}(w) \mathcal{F}_{i j}^{\mu \nu}(w) \delta \kappa_{j \nu}(w) .
$$

The thermodynamic fluctuations are encoded in the fluctuation matrix

$$
\mathcal{F}_{i j}^{\mu \nu}=\frac{1}{T}\left(\begin{array}{ccc}
\hat{u} \frac{\partial s}{\partial T} & \hat{u} \frac{\partial s}{\partial \mu_{B}} & s \hat{v}^{\nu} \\
\hat{u} \frac{\partial s}{\partial \mu_{B}} & \hat{u} \frac{\partial n}{\partial \mu_{B}} & n \hat{v}^{\nu} \\
s \hat{v}^{\mu} & n \hat{v}^{\mu} & -\hat{u}\left(T s+\mu_{B} n\right) g^{\mu \nu}
\end{array}\right)_{i j},
$$


with $\hat{u}=\hat{v}_{\mu} u^{\mu}$. As $\mathcal{F}(w)$ is local, so are the thermodynamic fluctuations. Assuming that the fluctuations are small, only Gaussian fluctuations contribute, and the only nontrivial correlator in Eq. (10) is the two-point function:

$$
\begin{aligned}
n_{12} \equiv & \left\langle\frac{d^{3} N_{\phi}}{d \mathbf{p}_{1}^{3}} \frac{d^{3} N_{\phi}}{d \mathbf{p}_{2}^{3}}\right\rangle_{c} \\
= & \frac{4}{(2 \pi)^{6}} \int d \Sigma^{\mu} \int \frac{d p_{1}^{0}}{2 \pi} \frac{d p_{2}^{0}}{2 \pi}\left(p_{1}\right)_{\mu}\left(\hat{v} \cdot p_{2}\right) \Theta\left(\breve{p}_{1}^{0}\right) \Theta\left(\breve{p}_{2}^{0}\right) \\
& \times\left.\left[\frac{\partial F_{\phi}\left(\breve{p}_{1}\right)}{\partial \kappa_{i}^{\rho}} \frac{\partial F_{\phi}\left(\breve{p}_{2}\right)}{\partial \kappa_{j}^{\sigma}}\right]\right|_{\bar{\kappa}}\left[\mathcal{F}_{i j}^{\rho \sigma}(w)\right]^{-1} .
\end{aligned}
$$

All other correlation functions can be obtained via Wick's theorem, as products of two-point functions. As before, we can evaluate this in the blast-wave approximation with the Wigner function of Eq. (3) and the spectral function of Eq. (6). To specify the thermodynamic quantities on the freeze-out surface, we use a hadron resonance gas model [31] which includes known particles and resonances with masses up to $2.6 \mathrm{GeV}$ [32].

In Fig. 3 we show the resulting connected two-point correlator as a function of transverse momenta in the moat regime, again with illustrative parameters $Z=-0.05$, $W=2.5 \mathrm{GeV}^{-2}$, and $m_{\text {eff }}=20 \mathrm{MeV}$. As before, we set $\phi_{p}$ and $y$ to zero and note that little changes qualitatively at nonzero rapidity. There is a clear enhancement of the fluctuations at a nonzero momentum, in contrast to the normal phase, where no such structure occurs. Comparing with the single-particle spectrum in Fig. 2, measuring the fluctuations through the two-particle correlation function yields a much greater enhancement in the moat regime, as compared with the normal phase. While our model is a gross simplification of the hadronic cocktail which contributes to a realistic simulation, any ordinary spectra will

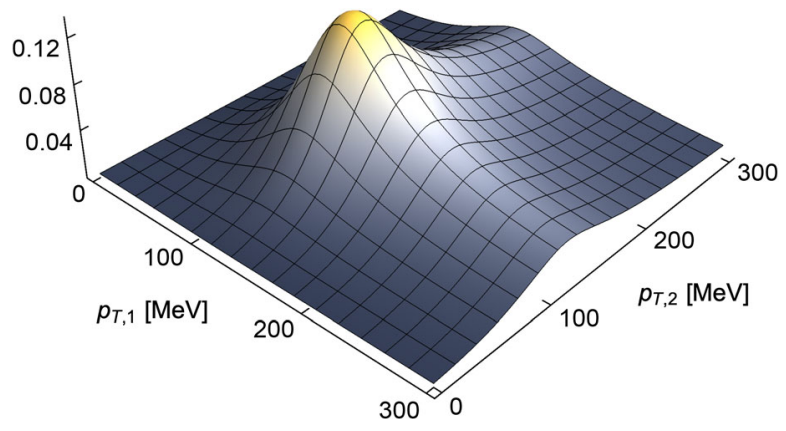

FIG. 3. In the moat regime, the connected two-particle correlation function normalized by the mean spectra, $n_{12} /\left(n_{1} n_{2}\right)$, Eqs. (4) and (14), as a function of $p_{T}$. The peak and the two ridges show that fluctuations are enhanced at a momentum related to the wave number of the spatial modulation of the moat regime. The correlation in the normal phase is basically flat and much smaller compared with the peak in the moat regime, by at least $\sim 10^{-2}$. uniformly peak at zero momentum, and yield a tiny and flat two-particle distribution, as compared to Fig. 3.

Conclusions.-We have shown how a moat regime leaves its imprint in particle spectra and their correlations. The key signature is that both show a characteristic peak at a nonzero momentum at the bottom of the moat. Thus, $p_{T}$-resolved measurements of these quantities in heavy-ion collisions at low energy are an efficient way of detecting moat regimes. While the formalism which we developed is general, we used simple models for the evolution of matter produced in heavy-ion collisions. Still, our results capture the essential physical features and therefore provide a first guide for how and where to look for novel phases in the QCD phase diagram. Experimentally, the main problem is to measure the particle spectrum at sufficiently low momentum. In the Beam Energy Scan II at RHIC, momenta down to $\sim 60 \mathrm{MeV}$ will be measured. Hopefully the novel signatures which we propose may appear.

A more detailed analysis will be presented separately [18].

We thank H. Caines, W. Christie, S. Floerchinger, E. Grossi, and L. Ruan for discussions and comments. This research was supported by the U.S. Department of Energy under Contract No. DE-SC0012704 and by BNL under the Lab Directed Research and Development program 18-036.

*rob.pisarski@gmail.com

†rennecke@bnl.gov

[1] K. Fukushima and T. Hatsuda, The phase diagram of dense QCD, Rep. Prog. Phys. 74, 014001 (2011).

[2] A. Bzdak, S. Esumi, V. Koch, J. Liao, M. Stephanov, and N. $\mathrm{Xu}$, Mapping the phases of quantum chromodynamics with beam energy scan, Phys. Rep. 853, 1 (2020).

[3] M. Buballa and S. Carignano, Inhomogeneous chiral condensates, Prog. Part. Nucl. Phys. 81, 39 (2015).

[4] G. Basar and G. V. Dunne, Self-Consistent Crystalline Condensate in Chiral Gross-Neveu and Bogoliubovde Gennes Systems, Phys. Rev. Lett. 100, 200404 (2008).

[5] G. Basar and G. V. Dunne, A twisted kink crystal in the chiral Gross-Neveu model, Phys. Rev. D 78, 065022 (2008).

[6] G. Basar, G. V. Dunne, and M. Thies, Inhomogeneous condensates in the thermodynamics of the chiral NJL(2) model, Phys. Rev. D 79, 105012 (2009).

[7] Speaking strictly the results derived only apply at infinite $N_{f}$. Because of the infrared fluctuations in two spacetime dimensions, at finite $N_{f}$ the $O(2)$ symmetry cannot spontaneously break; similarly, for a discrete symmetry at nonzero temperature. This is special to two spacetime dimensions, as in more than two spacetime dimensions, the spatially inhomogeneous condensate indicated by mean field theory does persist for either a discrete or $O(2)$ symmetry. There are numerous examples in condensed matter, such as smectic-C liquid crystals, etc., which confirm this. 
[8] We emphasize that this is different from the Landau-Peierls theorem, where fluctuations in transverse spatial direction around a one-dimensional inhomogeneous condensate lead to logarithmic divergences [9].

[9] L. Landau and E. Lifshitz, Statistical Physics, Part 1, Course of Theoretical Physics, Vol. 5 (ButterworthHeinemann, Oxford, 1980).

[10] R. D. Pisarski, F. Rennecke, A. Tsvelik, and S. Valgushev, The Lifshitz regime and its experimental signals, Nucl. Phys. A1005, 121910 (2021).

[11] R. D. Pisarski, A. M. Tsvelik, and S. Valgushev, How transverse thermal fluctuations disorder a condensate of chiral spirals into a quantum spin liquid, Phys. Rev. D 102, 016015 (2020).

[12] R. D. Pisarski, Remarks on nuclear matter: How an $\omega_{0}$ condensate can spike the speed of sound, and a model of Z(3) baryons, Phys. Rev. D 103, 071504 (2021).

[13] A. M. Tsvelik and R. D. Pisarski, Low energy physics of interacting bosons with a moat spectrum, and the implications for condensed matter and cold nuclear matter, arXiv: 2103.15835 .

[14] In the chiral limit, for three flavors the Goldstone bosons should disorder the chiral spiral into a quantum liquid of pions and kaons. What happens in QCD, when the strange quark is much heavier than the up and down quarks, is not obvious. The kaon fluctuations could either form a quantum liquid, like the pions, or a real kink crystal; see Ref. [11].

[15] W.-j. Fu, J. M. Pawlowski, and F. Rennecke, QCD phase structure at finite temperature and density, Phys. Rev. D 101, 054032 (2020).

[16] In Ref. [15] indications of a moat regime were found for $\mu_{B} \gtrsim 420 \mathrm{MeV}$ and $T \gtrsim T_{c}\left(\mu_{B}\right)$. In this case, $Z$ and $W$ in Eq. (2) parametrize the momentum dependence of quarkantiquark scattering in meson (specifically pion) exchange channels. Hence, moat regimes in the deconfined phase correspond to enhanced scattering at $p_{\min }$ in these channels. If this feature persists in the hadronic phase, it directly translates into a meson dispersion as in Eq. (2). However, this has not been analyzed in [15].

[17] F. Cooper and G. Frye, Comment on the Single Particle Distribution in the Hydrodynamic and Statistical Thermodynamic Models of Multiparticle Production, Phys. Rev. D 10, 186 (1974).

[18] R. D. Pisarski and F. Rennecke (to be published).

[19] E. Grossi, A. Soloviev, D. Teaney, and F. Yan, Transport and hydrodynamics in the chiral limit, Phys. Rev. D 102, 014042 (2020).
[20] A. Erschfeld, S. Floerchinger, and M. Rupprecht, General relativistic nonideal fluid equations for dark matter from a truncated cumulant expansion, Phys. Rev. D 102, 063520 (2020).

[21] E. Grossi, A. Soloviev, D. Teaney, and F. Yan, Soft pions and transport near the chiral critical point, Phys. Rev. D 104, 034025 (2021).

[22] E. Schnedermann, J. Sollfrank, and U. W. Heinz, Thermal phenomenology of hadrons from 200-A/GeV S+S collisions, Phys. Rev. C 48, 2462 (1993).

[23] W. Florkowski, Phenomenology of Ultra-Relativistic Heavy-Ion Collisions (World Scientific Publishing Company, Singapore, 2010).

[24] D. Teaney, The Effects of viscosity on spectra, elliptic flow, and HBT radii, Phys. Rev. C 68, 034913 (2003).

[25] R.-A. Tripolt, N. Strodthoff, L. von Smekal, and J. Wambach, Spectral functions for the quark-meson model phase diagram from the functional renormalization group, Phys. Rev. D 89, 034010 (2014).

[26] C. Jung, F. Rennecke, R.-A. Tripolt, L. von Smekal, and J. Wambach, In-medium spectral functions of vector- and axial-vector mesons from the functional renormalization group, Phys. Rev. D 95, 036020 (2017).

[27] R.-A. Tripolt, D. H. Rischke, L. von Smekal, and J. Wambach, Fermionic excitations at finite temperature and density, Phys. Rev. D 101, 094010 (2020).

[28] A. Andronic, P. Braun-Munzinger, K. Redlich, and J. Stachel, Decoding the phase structure of QCD via particle production at high energy, Nature (London) 561, 321 (2018).

[29] S. Zhang, Y. G. Ma, J. H. Chen, and C. Zhong, Beam energy dependence of Hanbury-Brown-Twiss radii from a blastwave model, Adv. High Energy Phys. 2016, 9414239 (2016).

[30] Private communication with S. Floerchinger (2016), and unpublished work presented by S. Floerchinger at the workshop "Functional Methods in Strongly Correlated Systems" in Hirschegg, Austria, on April 4, 2019.

[31] P. Braun-Munzinger, K. Redlich, and J. Stachel, Particle production in heavy ion collisions, Quark-Gluon Plasma 3, 491 (2003).

[32] P. A. Zyla et al. (Particle Data Group), Review of particle physics, Prog. Theor. Exp. Phys. 2020, 083 C01 (2020).

Correction: An erroneous statement in the Acknowledgments section has been removed. 